\title{
Effects of Self-Efficacy and Stigmatization when Managing Patients with Addiction and Substance Use Disorders
}

\author{
Catherine Spaccia \\ Dominican University of California
}

Introduction
- People who suffer with addiction are more likely to be
treated as outsiders, which result in social disadvantages
and maltreatment in a medical setting. This is because
substance abuse can be perceived as deviating from social
norms (Henderson \& Dressler, 2017).
- Higher post-secondary education on addiction could
reduce stigmatization and improve self-efficacy for better
healthcare outcomes. Previous research has revealed that
appropriate training is important when forming
nonjudgmental attitudes towards drug users (Baldwin et al.,
2006).
The purpose of this study is to address the gap between
post-secondary education, reducing stigmatization and
improving self-efficacy among healthcare professionals.

\section{Hypotheses}

Students with more education on addiction tend to have less stigmatization towards patients with addiction.

Students with higher self-efficacy tend to have less stigmatization towards patients with addiction.

Students with more education on addiction tend to have higher self-efficacy when managing patients with addiction.

\section{Measures}

Drug and Drug Users' Problems Perceptions Questionnaire (DDPPS; Puskar et al., 2013), a 22-item self-report Likert scale measured self-efficacy.

Drug Use Stigmatization Scale (DUSS; Palamar, 2011), a 7-item self-report Likert scale, measured stigmatization.

Level of education was measured using a question asking how many courses covering the topic at hand with four responses:

A - None (I have never taken a course that has addressed this topic)

- Little (I have taken a course that touched on this topic)

- Fair Amount (I have taken a full course on this topic)

- Substantial (I have taken multiple courses on this topic)

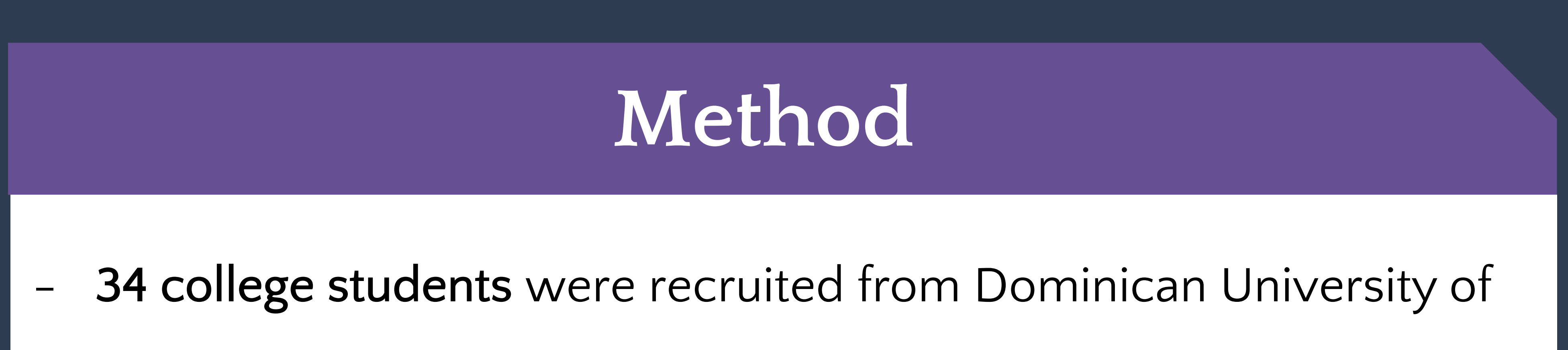

California (DUC). $87 \%$ were female; $13 \%$ were male; the mean age was $29.77(S D=13.11)$

Participants voluntarily completed an online survey, which included a combination of questions from two scales: DDPPS and DUSS, they indicated their level of education, along with demographic questions.

After completing the survey, students were thanked and provided a link to information about substance use disorders and addiction.

A Spearman rho and Pearson $r$ was used to calculate correlations between the variables of interest.

\section{Results}

When correlating level of education and stigmatization, there was no significant finding $\rho(30)=.134, p>.05$.

When correlating level of education and self-efficacy, there was a significant finding $\rho(30)=-.497, p=.004$.

When correlating stigmatization and self-efficacy, there was no significant finding

$$
r(30)=-0.197, p>.05
$$

A supplementary analysis found a significance between marijuana and higher levels of education on addiction $\rho(30)=.389, p=.028$.

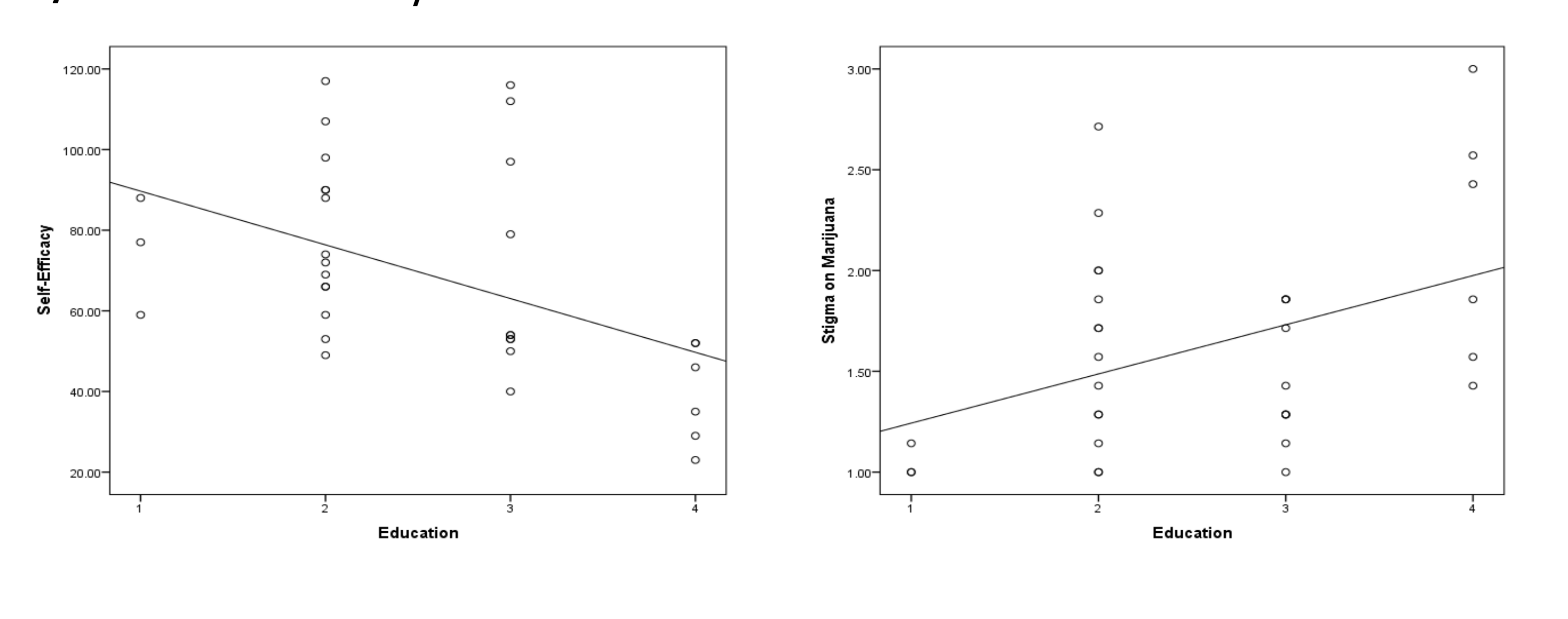

\section{Discussion}

It is important to note that education on addiction can influence a person's self-efficacy and stigmatization. This study's limitations were confined to DUC students in Marin County, leaving little room for a more culturally diverse research basis. Future studies can focus on what strategies are most effective for better healthcare outcomes for people who suffer with addiction, and use samples from other demographic backgrounds. 